\title{
Unexpected large transverse magneto-optic Kerr effect at quasi-normal incidence in magnetoplasmonic crystals
}

\author{
R. Cichelero ${ }^{1,}{ }^{*}$, M. A. Oskuei ${ }^{2}{ }^{\dagger}$, M. Kataja ${ }^{1}$, S.M. Hamidi ${ }^{2, \neq}$, G. Herranz ${ }^{1, \neq}$ \\ ${ }^{1}$ Institut de Ciència de Materials de Barcelona (ICMAB-CSIC), Campus UAB, 08193, Bellaterra, Catalonia, Spain \\ ${ }^{2}$ Laser and Plasma Research Institute, G. C., Shahid Beheshti University, Tehran 1983963113, Iran
}

‡Corresponding authors: $\underline{m}$ hamidi@sbu.ac.ir, gherranz@icmab.cat

We investigate the transverse magneto-optic Kerr effect (TMOKE) of magnetoplasmonic crystals grown on top of commercial optical disks. From full angle-resolved scans we can identify Wood's anomalies related to the excitation of plasmons of different orders. From these maps we also detect a wide range of wavelengths and angles of incidence for which the TMOKE signal is increased due to the interaction of light with surface propagating plasmons. Remarkably, conditions are established for unexpectedly large responses at quasi-normal incidence, where, by fundamental symmetry reasons, the intrinsic TMOKE should be vanishingly small. The key towards this unexpected outcome is to engineer the geometry of magnetoplasmonic crystals, so that firstorder plasmon dispersion lines run up towards quasi-normal angles of incidence. These results provide general rules for magneto-optic enhancement and, in particular, show the potential of standard commercial disks as platforms for enhanced magneto-optic devices.

\section{Highlights}

- Use of full angle-resolved reflectance spectroscopy to reveal band structures in the reciprocal space.

- Observation of unexpectedly large transverse magneto-optical responses at quasi-normal incidence.

- The phenomenon is observed in magnetoplasmonic crystals defined on top of off-the-shelf digital optical media.

Keywords: Magnetoplasmonics, magneto-optics, optic storage

${ }^{*}$ R.C. and M.A.O. contributed equally to this work. 


\section{Introduction.}

Surface plasmons are collective oscillations of electric charges at metal/dielectric interfaces coupled to electromagnetic waves. These plasmonic modes confine the electromagnetic fields to subwavelength volumes, so that the local intensity of the fields is increased enormously. This property enables a large magnification of the interaction of light with matter and is exploited, e.g., for the detection of single molecules in SERS (surface-enhanced Raman spectroscopy) ${ }^{1,2}$. As the properties of surface plasmons (resonance frequency, wavevector) are extremely sensitive to the changes of permittivity of the material surrounding the metal, they are nowadays widely used in applications for sensing ${ }^{3,4}$.

The enhancement of light-matter interactions made possible by plasmonics has also been used to boost up magneto-optical responses ${ }^{5}$. This is of particular interest for applications in integrated nanophotonics, as it requires materials with largely enhanced responses, so that they retain a strong magneto-optic activity even when downscaled to nanometric thickness. Along this line, it has been shown that the incorporation of ferromagnetic thin layers into metal/dielectric systems supporting plasmonic resonances can lead up to large increases of magneto-optic Kerr 6, 7, 8 , Faraday ${ }^{9,10,11,12}$ and transverse effects ${ }^{13,14,15}$. These enhanced magneto-optic responses may have potential technological impact for label-free detection using magnetoplasmonic sensors $16,17,18$. Alternatively, the combination of plasmonic nanostructures with magneto-optic materials paves the way to the development of ultracompact nonreciprocal photonic devices, of interest for integrated Faraday isolators and rotators in photonic circuitry 12,19, $20,21$.

Furthermore, in heat-assisted magnetic recording, plasmonic nanoantennas are used to locally heat the recording medium to reduce its coercivity, which enables reducing the size of individual bits and achieving higher storage densities ${ }^{22,23}$. The possibility to fabricate nanoantennas out of the recording media itself has also been investigated ${ }^{24}$. All-optical magnetic recording, where ultrashort, intense light pulses are used to control magnetization via the inverse Faraday and magneto-optical Kerr effects, can offer interesting possibilities when combined with the ability of plasmon resonances to confine and enhance electric fields ${ }^{25}$.

These developments have spurred an interest to design and fabricate devices based on magnetoplasmonic structures ${ }^{6,26}$. This, in turn, has urged the need to develop inexpensive, reproducible and largely-scalable methods for the development of magnetoplasmonic nanodevices. In this regard, an interesting platform is provided by off-the-shelf commercial digital disks, whose surface is covered by periodic spirally patterned tracks. They naturally act as sub-wavelength optical gratings, able to provide the necessary phase-match condition to couple light to plasmons ${ }^{27}$. Indeed, over the recent years, digital disk media, such as CD, DVD or Blu-ray have been used as platforms for plasmonic systems of use for biosensing ${ }^{28,29}$ or photocatalytic applications ${ }^{30}$. Interestingly, a large increase of the transverse magneto-optic Kerr (TMOKE) signal -associated to the excitation of plasmons- was observed at large angles of incidence $\left(\Theta \geq 45^{\circ}\right)$ in magnetoplasmonic crystals grown on commercial digital disks ${ }^{31}$. 


\section{Experimental methods.}

\subsection{Sample preparation.}

In regard to its technological potential, it is useful to explore the TMOKE response on commercial disk gratings over a wide range of angles of incidence, including values approaching the normal to the surface. This is an important aspect, as this geometry is used for readout in optic storage media, where reflected light is collected by high-numerical-aperture optics. With this purpose in mind, we performed full angle-resolved reflectance and magneto-optical experiments to map the TMOKE response of magnetoplasmonic systems fabricated on top of a digital DVD disk. To expose the surface grating, the first step was to remove the hard protective layer from the surface of small pieces (area ca. 10×10 $\mathrm{mm}^{2}$ ) cut out from a polycarbonate DVD disk by cleansing in alcohol, thus revealing a grating with periodicity $\delta=740 \mathrm{~nm}$, width $w=500 \mathrm{~nm}$, base $s=240 \mathrm{~nm}$ and depth $d=120 \mathrm{~nm}$ (Fig. 1(a)). Afterwards, the exposed disk grating was covered with $\mathrm{Cr}(4 \mathrm{~nm}) /$ $\mathrm{Au}(16 \mathrm{~nm})$ / [Co $(14 \mathrm{~nm})$ / Au (16 nm)] x4 / Co $(14 \mathrm{~nm})$ / Au (7 nm) coating by e-beam evaporation, being the total nominal thickness of the nanowires $t=161 \mathrm{~nm}$. The presence of cobalt results in higher losses thus creating a trade-off between the magneto-optical activity and plasmon propagation distance. We performed an optimization procedure using a commercially available Lumerical FDTD software to find the used gold and cobalt layer thicknesses. The magneto-optical activity in the FDTD was implemented by a coordinate rotation of the permittivity of the Co layer following the procedure outlined in Ref. 32. The numbers obtained via our method are in agreement with the results of earlier works aimed at optimizing magnetoplasmonic gold/cobalt multilayers ${ }^{33}, 34$. Magnetic hysteresis loops measured by SQUID magnetometry indicate that, as expected, the easy axis for magnetization is along the in-plane direction of the grating (the loops are shown in Fig. 3(b)-(d). Fig. 1(b) shows a representative SEM image of one of the gratings exposed on the surface of a DVD disk after the growth of the magnetic multilayered stack, indicating that the corrugation of the polycarbonate disk is preserved after the e-beam deposition.

\subsection{Optical characterization: angle-resolved reflectance spectroscopy.}

Fig. 1(c) shows a schematic depiction of the experimental setup used to visualize the emission of light diffracted from the gratings. Light coming from the source was filtered through a monochromator to select specific wavelengths within the visible range and then was collimated by a 10x objective lens (Obj1) then directed onto the sample through a second objective lens (Obj2) and a beamsplitter. Two different objective lenses where used, a $63 x$ oil immersion with numerical aperture N.A. $=1.4$ and a $50 \times$ N.A.= 0.75 lens. Before impinging on the specimen, a polarizer was used to set the polarization of the incoming light either as transverse magnetic (TM) or transverse electric (TE). After reflection from the sample, the beam was projected with the help of a flipmirror towards one of the two cameras. One was used for focusing purposes (CCD) to collect the near field image and the second one (sCMOS) was placed in the Fourier plane to collect the far field intensity for each wavelength and magnetic state of the sample. 
In our experiments, the beam illuminates the full back aperture of the objective lens (Obj2 in Fig. 1(c)). This way, light focused on the sample forms a constricted solid cone spanning a range of incident angles $\left(\Delta \Theta_{i n c} \approx \pm 52^{\circ}\right.$ for immersion oil and $\Delta \Theta_{i n c} \approx \pm 44^{\circ}$ for air) and covering the whole range of azimuthal $(\psi)$ angles. Fourier space maps are then obtained at every wavelength by plotting in polar coordinates the intensity of the light reflected for each value of $\Theta_{i n c}$ and $\psi$. Subsequently, angle-resolved maps are obtained by selecting an appropriate value of $\psi$ in every Fourier map (the data analysis is made using $\psi$ in range of 3 to -3 degrees, in the vicinity of the TM axis), corresponding to a given polarization state (see the sketch of Fig. 1(d) and Supplementary Information). For that purpose, after calibration using a commercial grating, space coordinates in the Fourier map along $\psi$ are converted to angles of incidence in the angle-resolved maps. The procedure is repeated over the whole range of wavelengths to obtain a full angle-resolved scan, where either reflectance or TMOKE are mapped out as a function of wavelength and angle of incidence.

\section{Results and discussion.}

\subsection{Unexpectedly large TMOKE at quasi-normal incidence.}

Fig. 2(a) shows the TMOKE Fourier space maps acquired at $\lambda=800 \mathrm{~nm}$ with the sample in air (measurements carried out using immersion oil are shown Supplementary Fig S1). Angle-resolved reflectance maps, where the sample intensity is normalized to the reflectance of an aluminum mirror, are shown in Fig. 2(b). Across these maps colored dotted lines indicate the dispersion curves of surface plasmons of different orders, for which the phase-matching condition is fulfilled, i.e., $k_{S P}=k_{x} \pm \eta G$. In this expression $G=\frac{2 \pi}{\delta} n$ is the reciprocal vector, $\eta= \pm 1, \pm 2, \pm 3, \ldots$ is the order and $k_{S P}, k_{x}$ are, respectively, the wavevectors of plasmons and the projection of the incident radiation wavevector along the normal $n$ to the grating structure. The wavevector of plasmons is calculated from $k_{S P}=\frac{2 \pi}{\lambda_{S P P}}=2 \pi\left(\lambda \sqrt{\frac{\epsilon_{d} \epsilon_{m}}{\epsilon_{d}+\epsilon_{m}}}\right)^{-1}$, where $\lambda$ is the wavelength of the incident light and $\epsilon_{d}$ and $\epsilon_{m}$ are, respectively, the permittivity of the dielectric medium (either air $\sqrt{\epsilon_{d}}=1$ or immersion oil $\sqrt{\epsilon_{d}} \approx 1.45$ ) and the effective permittivity of the magnetic metallic multilayer, which has been obtained from ellipsometry measurements. In the maps, the excitation of plasmons of orders $\eta= \pm 1$ is revealed as a dip in reflectance, known as Wood's anomalies ${ }^{35},{ }^{36}$, along the lines predicted for their dispersion curves (Fig. 2(b)). On the contrary, plasmons of higher orders cannot be discerned; plausibly, the coupling of light to plasmons is weaker as $\eta$ increases, becoming barely visible in the reflectance maps. Yet, as we will see below, the presence of SPPs with $\eta>1$ is evident in angle-resolved TMOKE maps.

The TMOKE signal is defined as the magnetic-field dependent variation of the intensity of light reflected off the grating structure and it's given by $\tau=\frac{I\left(H^{+}\right)-I\left(H^{-}\right)}{I(H=0)}$, where $I(H=0), I\left(H^{+}\right)$and $I\left(H^{-}\right)$are, respectively, the intensities for magnetic fields above saturation $(|H| \gtrsim 150 \mathrm{Oe})$ and 
$I(H=0)$ is defined as the average of the absolute value of $I\left(H^{+}\right)$and $I\left(H^{-}\right)$over many hysteretic cycles. The resultant angle-resolved TMOKE maps are displayed in Fig. 2(c). In these images we see that the magnitude of the TMOKE signal varies greatly as a function of wavelength and incidence angle. As expected from fundamental symmetry arguments, the TMOKE activity is weak for light incident close to the normal, while it increases for larger angles of incidence and shorter wavelengths. Yet, the weakness of the TMOKE signal at longer wavelengths and small angles of incidence is conspicuously broken along the lines following the SPP resonances, superimposed as colored dotted lines in the maps shown in Fig. 2(c), resulting in distinguishable anomalies in the TMOKE spectrum. In particular, we observe that the SPP lines influence the sign and the magnitude of the TMOKE effect and create bands of surprisingly high magneto-optical activity, which are discussed in the following.

To begin with, the profile data shown in Figure 2(d) demonstrates that the magneto-optical signal is increased up to $|\tau| \simeq 5 \times 10^{-3}$ along the plasmon dispersion lines, particularly along the firstorder $\eta= \pm 1$ lines. Interestingly, these lines and the corresponding enhanced TMOKE signal run up towards normal incidence at wavelengths $\lambda \approx 760 \mathrm{~nm}$. This is an unexpected result, since for smooth magnetic films TMOKE at normal incidence is strictly zero due to symmetry principles. Yet, our results demonstrate that the analysed magnetoplasmonic crystal exhibits $|\tau| \simeq 5 \times 10^{-3}$ at angles as low as $\theta \approx 1.3^{\circ}$ (Fig. 2(d)). We stress that this value is significantly above the largest intrinsic TMOKE $\left(|\tau| \leq 10^{-3}\right)$ in unstructured magnetic materials, which is only obtained at much larger angles of incidence. The key to this surprisingly large signal at quasi-normal incidence is to engineer the geometric parameters of the magnetoplasmonic crystal so that first-order plasmon lines approach normal incidence for some range of wavelengths. The plasmon line order is essential, since the TMOKE enhancement at higher-orders is smaller, as shown by our experiments in index oil (Supplementary Fig. S1). This observation is undoubtedly of high interest for applications that require sensing optical responses along the normal to the surface, analogous to the readout in optic storage media.

\subsection{Origin of the plasmon-enhanced TMOKE.}

We discuss next a few aspects of the observed magneto-optical enhancement that reveal valuable information about the origin of the phenomenon. In the first place, we note that the angleresolved maps of Fig. 2(c) show that the enhanced TMOKE signal around the plasmon lines has the expected derivative shape ${ }^{31}$, where the response undergoes a swift sign reversal and crosses zero at the SPP resonance at $\lambda \approx 640 \mathrm{~nm}$. This is a well-known result in the literature, where the observed line-shape is caused by a shift in the SPP energy as a function of the magnetization of the grating $37,38,39,40$.

Additionally, we note that the plasmon-induced increase of TMOKE is not discernible at short wavelengths and large angles of incidence. The last observation may be due, at least partially, to the fact that $\eta= \pm 2$ plasmons appear at large angles of incidence, where the intrinsic TMOKE 
signal is significant, thus masking out any effect of those plasmons on TMOKE. This conclusion is supported by measurements carried out in index matching oil with index $n=1.45$, where the grating exhibits a clear TMOKE feature at $\eta= \pm 2$ (Supplementary Fig S1). The TMOKE features are clearly discernible despite the absence of corresponding features in the Fourier space reflectance map. However, in contrast to the results on air at $\eta= \pm 1$, the TMOKE features at $\eta= \pm 2$ disappear at small angles of incidence (Supplementary Fig S1). We believe that the reason for this apparent disappearance of TMOKE is that the intrinsic response, i.e., the response of the unpatterned material in the absence of any plasmons, is vanishingly small as the angle of incidence is decreased. Thus, assuming that the enhancement factor is constant, below a certain threshold value for the angle of incidence, the TMOKE signal is too small to be observed, even in the presence of plasmons. Note that, the enhancement due to $\eta= \pm 1$ plasmons is observed at any value of the angle of incidence. The difference between $\eta= \pm 1$ and $\eta= \pm 2$ plasmons is that the enhancement factor is larger for the former, enabling the observation of the TMOKE signal even at low angles of incidence.

Before concluding, we note that the coupling between in-plane and out-of-plane localized plasmons in the metallic nanowires of the gratings may play a role in the enhancement of TMOKE at short wavelengths and large angles of incidence. More specifically, although angle-resolved spectroscopy maps do not show any clear evidence of localized plasmons with in-plane electric fields perpendicular to the nanowires axes (Supplementary Fig S2), we cannot exclude that out-ofplane localized plasmons are coupled to in-plane excitations, as suggested by K. Lodewijks et al ${ }^{41}$. In this respect, we note that, while the width of the nanowires is in the order of $500 \mathrm{~nm}$, which is too large to excite localized plasmons in the visible, the nominal thickness of the nanowires is significantly smaller ( $161 \mathrm{~nm}$ ). Although this is still too large to excite out-of-plane localized plasmons in the visible, we cannot exclude completely an eventual interplay between in-plane and out-of-plane plasmons that could play some role in the enhancement of TMOKE in the range of short wavelengths and large angles of incidence.

\section{Summary and conclusions.}

Summing up, we have investigated the transverse magneto-optic Kerr effect for light incident on magnetic metallic multilayers deposited on top of the grating structure of commercial disks. Wood's anomalies, related to the excitation of plasmons, are distinguishable in angle-resolved reflectance maps. It is shown that the TMOKE signal is visibly enhanced around the dispersions curves predicted for plasmons of different orders; the increase in signal is readily noticeable for low-order plasmons. Interestingly, conditions for plasmon-assisted TMOKE responses can be found for a wide range of angles of incidence, including quasi-normal incidence, where intrinsic TMOKE is absent. These results show that proper engineering of magnetoplasmonic crystals on top of massproduced standard commercial disks hold promise as platforms for magneto-optic devices. 
Acknowledgements. This work was supported by the Spanish MAT2017-85232-R (AEI/FEDER,UE), MAT2014-56063-C2-1-R, Severo Ochoa SEV-2015-0496 grant, the Generalitat de Catalunya (2017 SGR 1377). M.A.O. acknowledges financial support from the Ministry of Science, Research and Technology of Iran. R.C acknowledges support from CNPq Brazil. 


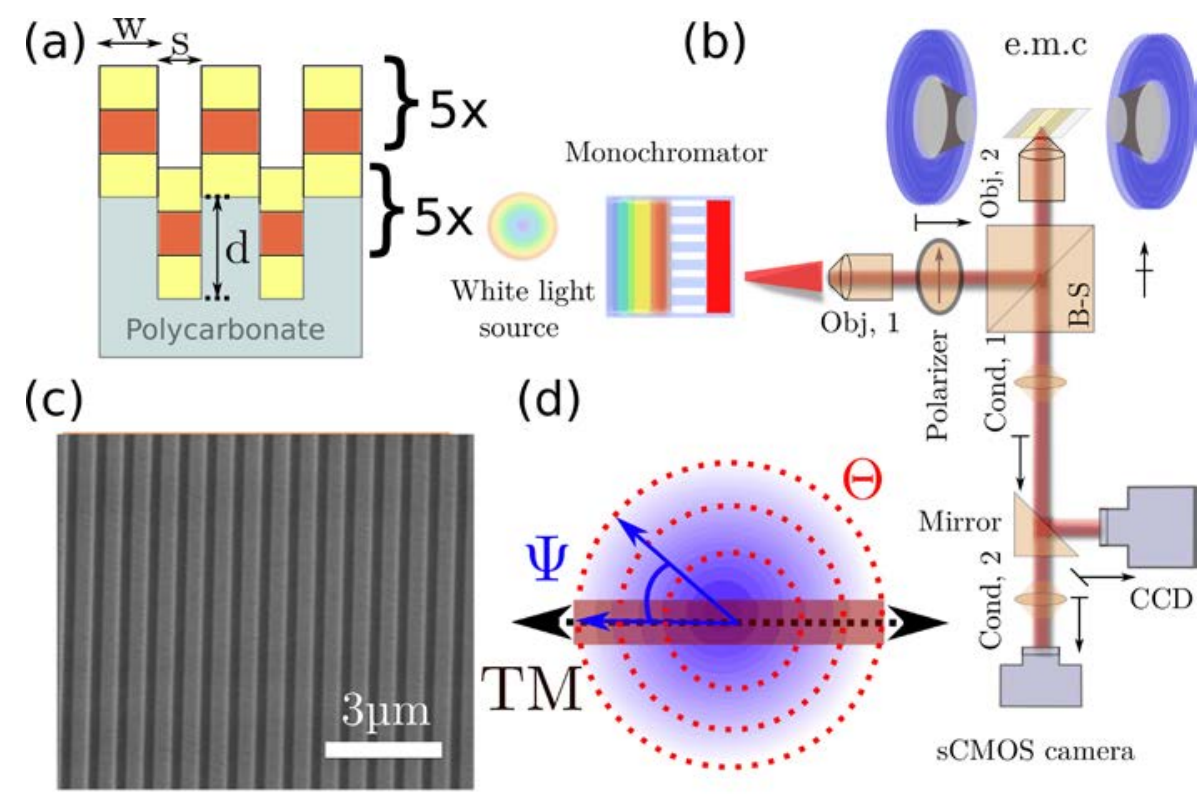

FIG 1. (a) Cross-sectional sketch of the magnetic metallic with $\mathrm{Cr}(4 \mathrm{~nm}) / \mathrm{Au}(16 \mathrm{~nm}) /$ [Co (14 nm) / Au (16 nm)] x4 / Co (14 nm) / Au (7 nm) multilayered stack on top of the corrugated surface of the polycarbonate disk, where $w, s$ and $d$ are the grating width, base and depth. (b) Schematics of the optical setup used to obtain Fourier and full angle-resolved reflectance maps. (c) Scanning electron microscope image of the surface of the disk covered by the multilayered stack. (d) Depiction of the back aperture of the focusing objective lens. Circular dashed lines designate loci of constant angle of incidence $(\Theta)$. The values of the azimuthal angle range within $\Psi \in[0,2 \pi]$ at a given dashed line (constant $\Theta$ ). The projection along the shaded strip designates a set of $\Psi$ and $\Theta$ values that correspond to the initial TM-polarized light plane (see also Supplementary Information). 
(a)

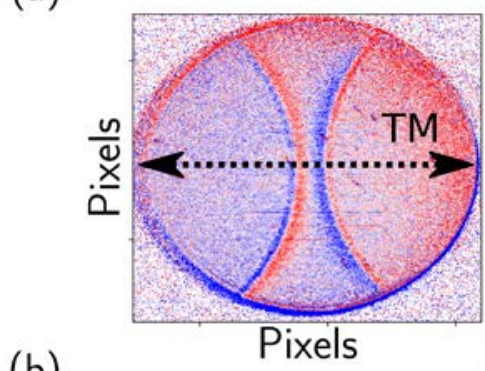

(b)

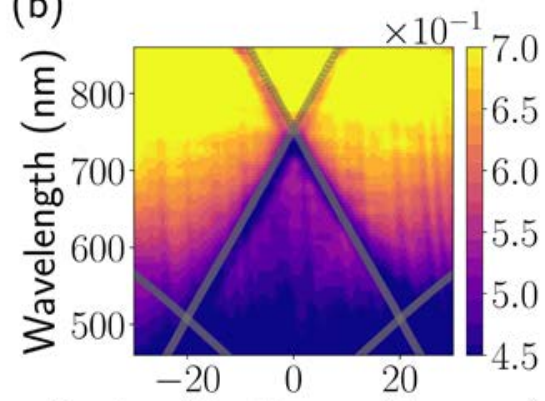

Angle of incidence (degrees) (c)

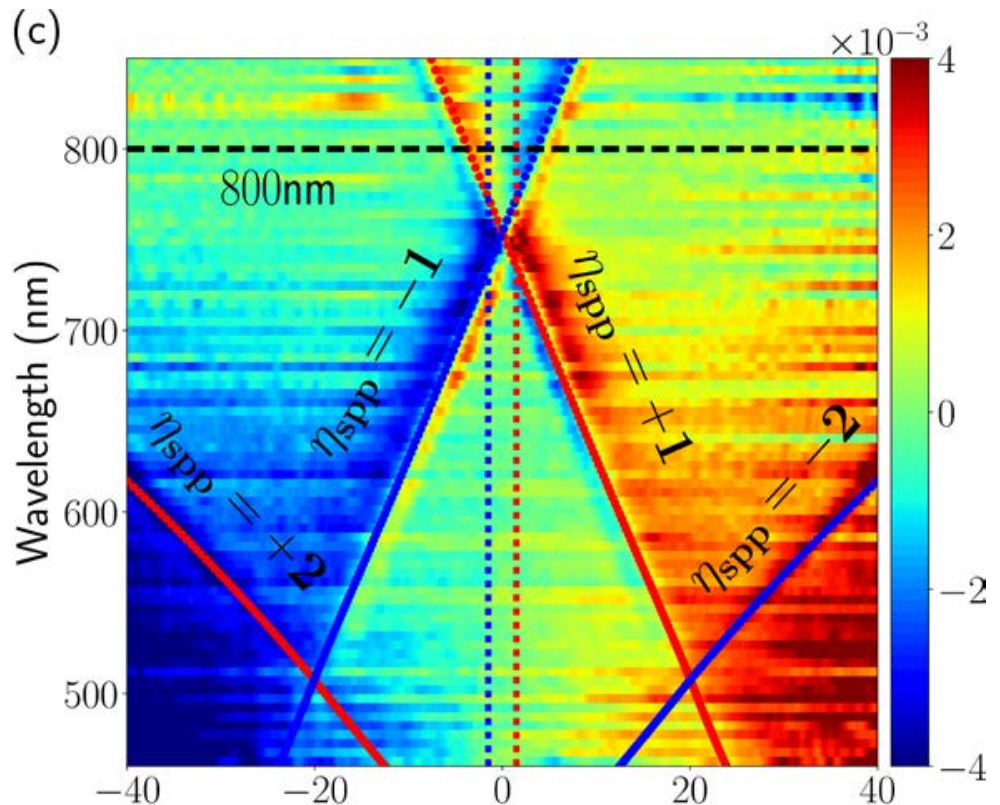

Angle of incidence (degrees)

(d)

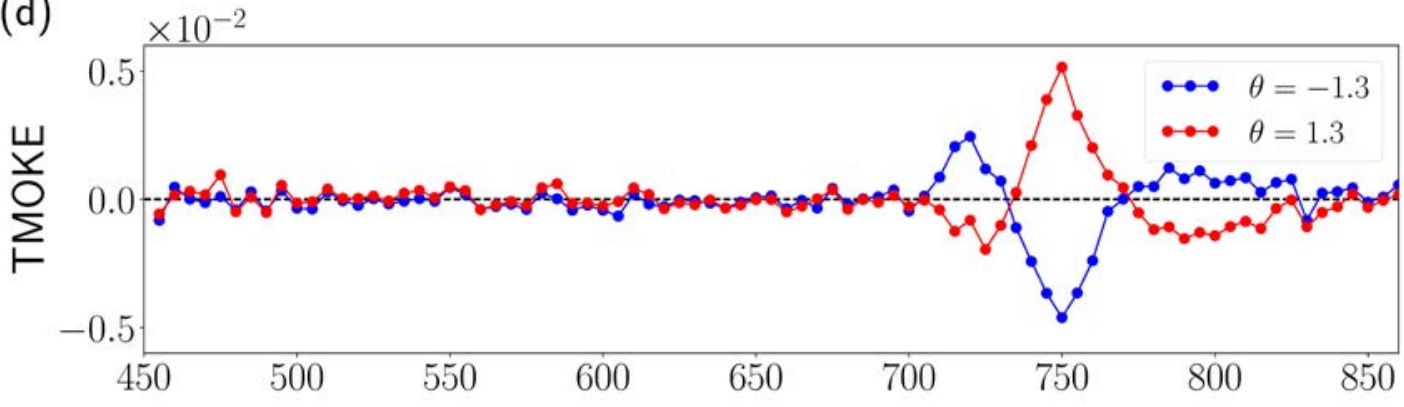

FIG 2. (a) TMOKE Fourier map obtained for air, at a wavelength $\lambda=800 \mathrm{~nm}$. The dotted horizontal arrows indicate the regions where the azimuthal angle $\Psi$ corresponds to TM-polarized light. A description of the procedure to obtain the Fourier maps is described in the main text. (b) Full angle-resolved reflectance maps for TM-polarized incident light, with the predicted plasmon lines of different orders superimposed as dotted lines. (c) Angle-resolved TMOKE maps. Plasmon dispersion curves are plotted with labels indicating their order index. The dashed horizontal line $(\lambda=800 \mathrm{~nm})$ indicates scan profiles obtained from TMOKE Fourier maps of panel (a) measured at positive and negative saturation magnetic fields. The full map shown in (c) is obtained by adding up the profiles obtained at all wavelengths, with a step $\Delta \lambda=5 \mathrm{~nm}$. (d) Cross-sectional TMOKE plots, acquired from the dashed vertical lines $\left(\theta=+1.3^{\circ}\right.$ and $\left.\theta=-1.3^{\circ}\right)$. 
(a)

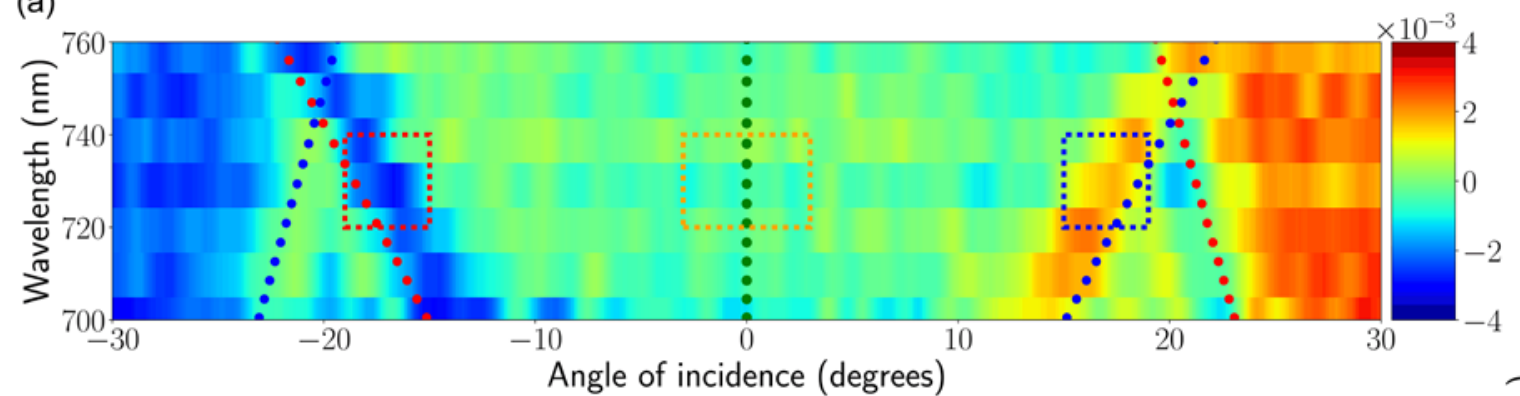

(b)

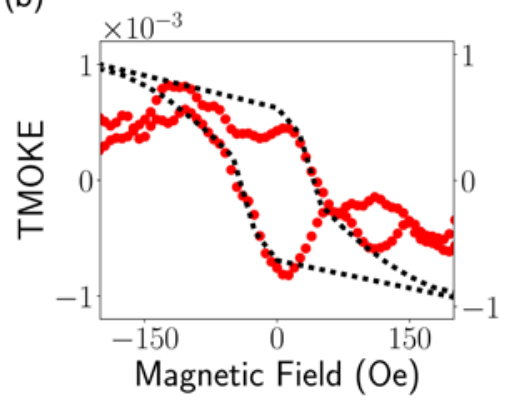

(c)

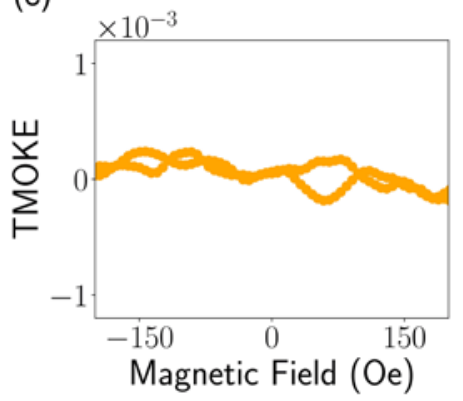

(d)

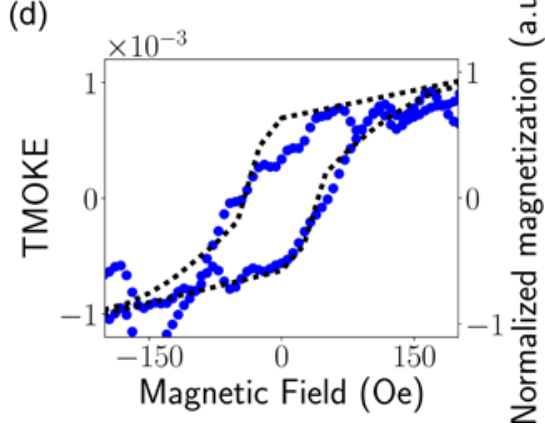

FIG. 3. (a) Selected area of the full angle-resolved TMOKE map (sample in oil), where the effect of first-order $\eta= \pm 2$ plasmons is observed. The colored squares in the map indicate the regions where the TMOKE signal was averaged to obtain the hysteresis loops displayed as colored dotted lines in the bottom (b), (c) and (d) panels. Magnetic hysteresis loops measured by SQUID are also displayed in black dotted curves. 


\section{REFERENCES}

${ }^{1}$ D. Cialla-May, X.-S. Zheng, K. Weber, and J. Popp, Chem. Soc. Rev. 46, 3945-3961 (2017).

${ }^{2}$ S. Schlücker, Angew. Chem. Int. Ed. Engl. 53, 4756-4795 (2014).

${ }^{3}$ B. Sepúlveda , A. Calle , L. M. Lechuga , and G. Armelles , Opt. Lett. 31 , 1085 (2006).

${ }^{4}$ M. Bauch, K. Toma, M. Toma, Q. Zhang, and J. Dostalek, Plasmonics 9,781-799 (2014).

${ }^{5}$ G. Armelles, A. Cebollada, A. García-Martín, and M. Ujué González, Adv. Optical Mater. 1, 10-35 (2013).

${ }^{6}$ M.Rubio-Roy, O. Vlasin, O. Pascu, J.M. Caicedo, M. Schmidt, A.R. Goñi, N.G. Tognalli, A. Fainstein, A. Roig, G. Herranz, Langmuir 28, 9010-9020 (2012).

7J. C. Banthí, D. Meneses-Rodríguez, F. García, M. U. González, A. García-Martín, A. Cebollada, and G. Armelles, Adv. Mater. 24, OP36-41 (2012).

${ }^{8}$ M. Kataja, T. K. Hakala, A. Julku, M. J. Huttunen, S. van Dijken, and P. Törmä, Nat. Commun. 6, 7072 (2015).

9J. Y. Chin, T. Steinle, T. Wehlus, D. Dregely, T. Weiss, V. I. Belotelov, B. Stritzker, and H. Giessen, Nat. Commun. 4, 1599 (2013).

${ }^{10}$ A. N. Kuzmichev, L. E. Kreilkamp, M. Nur-E-Alam, E. Bezus, M. Vasiliev, I. A. Akimov, K. Alameh, M. Bayer, and V. I. Belotelov, Materials 8, 3012-3023 (2015).

${ }^{11}$ B. Caballero,A. García-Martín, and J. C. Cuevas, Optics Express 23, 22238- 22229 (2015).

${ }^{12}$ D. Floess, M. Hentschel, T. Weiss, H.-U. Habermeier, J. Jiao, S. G. Tikhodeev, and H. Giessen, Phys. Rev X 7, 021048 (2017).

${ }^{13}$ E. Ferreiro-Vila, J. B. González-Díaz, R. Fermento, M. U. González, A. García-Martín, J. M. GarcíaMartín, A. Cebollada, and G. Armelles, D. Meneses-Rodríguez, and E. Muñoz Sandoval, Phys. Rev. B 80, 125132 (2009).

${ }^{14}$ L. E. Kreilkamp, V. I. Belotelov, J. Y. Chin, S. Neutzner, D. Dregely, T. Wehlus, I. A. Akimov, M. Bayer, B. Stritzker, and Harald Giessen, Phys. Rev. X 3, 041019 (2013).

${ }^{15}$ L. Halagacka, M. Vanwolleghem, K. Postava, B. Dagens, and J. Pistora, Opt. Express 21, 2174121755 (2013).

${ }^{16}$ N. Maccaferri , K. E. Gregorczyk, Thales V.A.G. de Oliveira, M. Kataja, S. van Dijken, Z. Pirzadeh, A. Dmitriev, J. Åkerman, M. Knez, and P. Vavassori, Nat Comms. 6, 6150 (2015).

${ }^{17}$ B. Caballero, A. García-Martín, and J. C. Cuevas, ACS Photonics 3, 203-208 (2016). 
${ }^{18}$ J. Qin, Y. Zhang, X. Liang, C. Liu, C. Wang, T. Kang, H. Lu, L. Zhang, P. Zhou, X. Wang, B. Peng, J. Hu, L. Deng, and L. Bi, ACS Photonics 4, 1403-1412 (2017).

${ }^{19}$ M. Liu, and X. Zhang, Nat. Photonics 7, 429 (2013).

${ }^{20}$ A. F. Koenderink, A. Alù, and A. Polman, Science 348, 516 (2015).

${ }^{21}$ C. J. Firby, P. Chang, A. S. Helmy, and A.Y. Elezzabi, ACS Photonics 3, 2344-2352 (2016).

${ }^{22}$ W. A. Challener, C. Peng, A. V. Itagi, D. Karns, W. Peng, Y. Peng, X. Yang, X. Zhu, N. J. Gokemeijer, Y.-T. Hsia, G. Ju, R. E. Rottmayer, M. A. Seigler, and E. C. Gage, Nature Photonics 3, 220-224 (2009).

${ }^{23}$ M. H. Kryder, E. C. Gage, T. W. McDaniel, W. A. Challener, R. E. Rottmayer, G. Ju, Y.-T. Hsia, and M. F. Erden, Proc. IEEE 96, 1810 - 1835 (2008).

${ }^{24}$ M. Kataja, F. Freire-Fernández, J. P. Witteveen, T. K. Hakala, P. Törmä, and S. van Dijken, Appl. Phys. Lett. 112, 072406 (2018).

${ }^{25}$ T.-M. Liu, T. Wang, A.H. Reid, M. Savoini, X. Wu, B. Koene, P. Granitzka, C.E. Graves, D.J. Higley, Z. Chen, G. Razinskas, M. Hantschmann, A. Scherz, J. Stöhr, A. Tsukamoto, B. Hecht, A.V. Kimel, A. Kirilyuk, T. Rasing, and H.A. Dürr. Nano Letters 15, 6862-6868 (2015).

${ }^{26}$ S. M. Hamidi, H. Normhammadi, and M. M. Tehranchi, J. Optics and Laser Technology 49, 237242 (2013).

${ }^{27}$ S.A. Maier, Plasmonics: Fundamentals and Applications, Ed. Springer Science (2007).

${ }^{28}$ K. Bhatnagar, A. Pathak, D. Menke, P. V. Cornish, K. Gangopadhyay, V. Korampally, and S. Gangopadhyay, Nanotechnology 23, 495201 (2012).

${ }^{29}$ A. I. Radu, Ye. Ye. Ussembayev, M. Jahn, U. S. Schubert, K. Weber, D. Cialla-May, S. Hoeppener, A. Heisterkampe, and J. Popp, RSC Adv. 6, 44163 (2016).

${ }^{30}$ S. Nootchanat, H. Ninsonti, A. Baba, S. Ekgasit, C. Thammacharoen, K. Shinbo, K. Katoa, and F. Kanekoa, Phys.Chem.Chem.Phys. 16, 24484 (2014).

${ }^{31}$ A. A. Grunin, A. V. Chetvertukhin, T. V. Dolgova, A. A. Ezhov, and A. A. Fedyanin, J. Appl. Phys. 113, 17 A946 (2013).

${ }^{32}$ https://kb.lumerical.com/en/other_application_magneto-optical_kerr_effect.html.

33 D. Regatos, B. Sepúlveda, D. Fariña, L. G. Carrascosa, and L. M., Optics Express 19, 8336-46 (2011).

${ }^{34}$ C. Aurelio Herreño-Fierro and E. J. Patiño, Physica Status Solidi (B) 252, 316-22 (2015). 
35 Maystre D. (2012) Theory of Wood's Anomalies. In: Enoch S., Bonod N. (eds) Plasmonics. Springer Series in Optical Sciences, vol 167. Springer, Berlin, Heidelberg

${ }^{36}$ R. Petit (ed.), Electromagnetic Theory of Gratings. Topics in Current Physics (Springer, Berlin, 1980)

${ }^{37}$ V. I. Belotelov, I. A. Akimov, M. Pohl, V. A. Kotov, S. Kasture, A. S. Vengurlekar, A. V. Gopal, D. R. Yakovlev, A. K. Zvezdin, and M. Bayer, Nature Nanotechnology 6, 370-376 (2011).

${ }^{38}$ A. V. Chetvertukhin, A.Grunin, A.V.Baryshev, T.V.Dolgova, H.Uchida, M.Inoue, and A.A. Fedyanin, J. Magn. Magn. Mater. 324, 3516-3518 (2012).

39 J. J. Brion, R. F. Wallis, A. Hartstein, and E. Burstein, Phys. Rev. Lett. 28, 1455-1458 (1972).

${ }^{40}$ M. S. Kushwaha, and P. Halevi, Phys. Rev. B 36, 5960-5967 (1987).

${ }^{41}$ K. Lodewijks, N. Maccaferri, T. Pakizeh, R. K. Dumas, I. Zubritskaya, J. Åkerman, P. Vavassori, and A. Dmitriev, Nano Lett. 14, 7207-7214 (2014). 